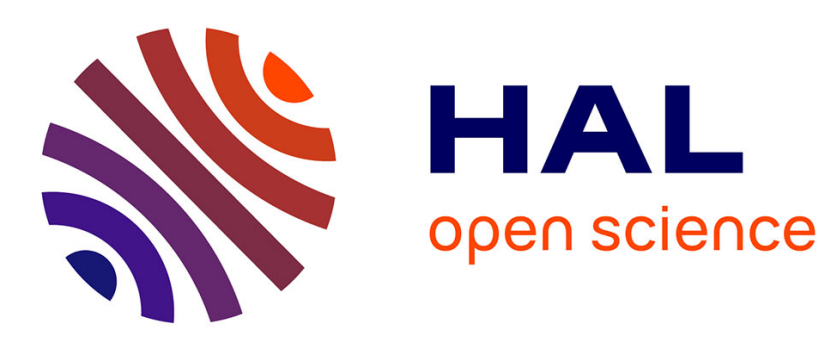

\title{
Comportement local moyen de la fonction de Brjuno
} Michel Balazard, Bruno Martin

\section{To cite this version:}

Michel Balazard, Bruno Martin. Comportement local moyen de la fonction de Brjuno. Fundamenta Mathematicae, 2012, 218 (3), p. 193-224. 10.4064/fm218-3-1 . hal-00723616

\section{HAL Id: hal-00723616 https://hal.science/hal-00723616}

Submitted on 11 Aug 2012

HAL is a multi-disciplinary open access archive for the deposit and dissemination of scientific research documents, whether they are published or not. The documents may come from teaching and research institutions in France or abroad, or from public or private research centers.
L'archive ouverte pluridisciplinaire HAL, est destinée au dépôt et à la diffusion de documents scientifiques de niveau recherche, publiés ou non, émanant des établissements d'enseignement et de recherche français ou étrangers, des laboratoires publics ou privés. 


\title{
COMPORTEMENT LOCAL MOYEN DE LA FONCTION DE BRJUNO
}

\author{
MICHEL BALAZARD ET BRUNO MARTIN
}

\begin{abstract}
We describe the average behaviour of the Brjuno function $\Phi$ in the neighbourhood of any given point of the unit interval. In particular, we show that the Lebesgue set of $\Phi$ is the set of Brjuno numbers and we find the asymptotic behaviour of the modulus of continuity of the integral of $\Phi$.
\end{abstract}

\section{KEYWORDS}

Brjuno function, Brjuno numbers, Lebesgue set, Continued fractions. MSC classification : 26A27 (11A55)

\section{INTRODUCTION}

Désignons par $\lfloor u\rfloor$ la partie entière du nombre réel $u$, et par $\{u\}$ sa partie fractionnaire :

$$
\{u\}=u-\lfloor u\rfloor, \quad\lfloor u\rfloor \in \mathbb{Z}, \quad 0 \leqslant\{u\}<1 .
$$

Posons $X=] 0,1[\backslash \mathbb{Q}$. L'application

$$
\begin{aligned}
\alpha: X & \rightarrow X \\
x & \mapsto\{1 / x\}
\end{aligned}
$$

définit une transformation mesurable de $X$ muni de la tribu borélienne dans lui-même. Ryll-Nardzewski (cf. [12], Theorem 2, p.76) a observé que la mesure de probabilité

$$
\mu=\frac{d x}{(1+x) \log 2}
$$

est invariante* par $\alpha$ : la mesure image $\alpha \mu$ n'est autre que $\mu$.

En d'autres termes, si $f \in L^{1}(0,1)$, alors $f \circ \alpha$ appartient aussi à $L^{1}(0,1)$ et

$$
\int_{0}^{1} f(\alpha(x)) \frac{d x}{1+x}=\int_{0}^{1} f(x) \frac{d x}{1+x}
$$

Bruno Martin a bénéficié d'un financement par la fondation autrichienne FWF dans le cadre du projet S9605 de son réseau Analytic Combinatorics and Probabilistic Number Theory. Les auteurs remercient d'autre part le laboratoire Poncelet (CNRS-Université Indépendante de Moscou) pour son accueil lors de la préparation de cet article.

*. On trouve une assertion équivalente dans l'article de Doeblin [3], p.357. 
Nous définissons les fonctions itérées de $\alpha$ en posant $\alpha_{0}(x)=x$ et pour $k \geqslant 1, \alpha_{k}(x)=\alpha\left(\alpha_{k-1}(x)\right)$. Les fonctions $\alpha_{k}(k \geqslant 0)$ sont définies sur $X$, à valeurs dans $X$, et laissent la mesure $\mu$ invariante $^{\dagger}$.

Posons

$$
\Phi(x)=\sum_{k \geqslant 0} \alpha_{0}(x) \alpha_{1}(x) \cdots \alpha_{k-1}(x) \log \left(1 / \alpha_{k}(x)\right) .
$$

Cette série à termes positifs définit une fonction $\Phi: X \rightarrow] 0, \infty]$, appelée fonction de Brjuno ${ }^{\ddagger}$. On constate aisément que $\Phi$ satisfait à l'équation fonctionnelle

$$
\Phi(x)=\log (1 / x)+x \Phi(\alpha(x)) \quad(x \in X) .
$$

Les nombres irrationnels $x$ pour lesquels $\Phi(x)=\infty$, appelés nombres de Cremer, constituent un ensemble de mesure de Lebesgue nulle. On appelle nombres de Brjuno les nombres $x$ pour lesquels la série converge.

La fonction de Brjuno intervient dans l'étude des systémes dynamiques engendrés par les itérations d'une fonction holomorphe $f: \mathbb{C} \mapsto \mathbb{C}$. Citons en particulier un théorème de Yoccoz [14] : si $f^{\prime}(0)=e^{2 i \pi x}$ où $x \in X$, alors $f$ est localement linéarisable au voisinage de $z=0$ si, et seulement si $x$ est un nombre de Brjuno.

Marmi, Moussa et Yoccoz [8] ont entrepris l'étude de la régularité de la fonction de Brjuno et de certaines de ses variantes. Ils établissent notamment que $\Phi$ appartient à $L^{p}(0,1)$ pour tout $p \geqslant 1$ et, ce qui est une proprièté plus forte, qu'elle est à oscillation moyenne bornée :

$$
\sup \frac{1}{|I|} \int_{I}\left|\Phi(x)-\frac{1}{|I|} \int_{I} \Phi(t) d t\right| d x<\infty,
$$

où le supremum est pris sur tous les intervalles ouverts $I$ inclus dans $] 0,1[$, et où $|I|$ désigne la longueur de $I$.

Dans ce travail, nous caractérisons les points de Lebesgue de $\Phi$ : étant donnée une fonction $f$ localement intégrable sur un intervalle $J$ de $\mathbb{R}$, on dit que $x$ est un point de Lebesgue de $f$ si

$$
\frac{1}{h} \int_{x-h / 2}^{x+h / 2}|f(t)-f(x)| d t=o(1) \quad(h \rightarrow 0) .
$$

Un théorème classique de la théorie de l'intégrale de Lebesgue stipule que presque tout élément de $J$ est un point de Lebesgue de $f$ (cf. [13] Chapitre 7 par exemple). Notre résultat principal est le suivant.

$\dagger$. On peut montrer que $\alpha$ est une transformation mélangeante sur $(X, \mu)$. Cela permet de démontrer une assertion de Gauss dans une lettre à Laplace du 30 janvier 1812 (sur la convergence de la fonction de répartition de $\alpha_{k}$, par rapport à la mesure de Lebesgue, quand $k \rightarrow \infty$, cf. [5], p.371-374). Cette assertion de Gauss avait été démontrée pour la première fois par Kuzmin en 1928, cf.[7]. Le $\S 4$ de [1] expose clairement cette théorie.

†. Il s'agit ici d'une variante de la fonction définie par Yoccoz dans [14], pp. 11-16 : nous utilisons la théorie habituelle des fractions continues, alors que Yoccoz en considére une version légérement modifiée. 
Théorème 1. Les points de Lebesgue de la fonction $\Phi$ sont exactement les nombres de Brjuno.

Notre deuxième résultat est une description complète du comportement local de la fonction croissante et absolument continue $\Psi$ définie par

$$
\Psi(x)=\int_{0}^{x} \Phi(t) d t \quad(0 \leqslant x \leqslant 1) .
$$

Pour le formuler, nous prolongeons d'abord $\Phi$ aux nombres rationnels de l'intervalle ]0, $1[$. Si $r \in] 0,1\left[\cap \mathbb{Q}\right.$, on peut définir $\alpha_{k}(r)$ par itération de $\alpha$ tant que $k$ ne dépasse pas la longueur $K=K(r)$ de l'écriture de $r$ en fraction continue; nous appellerons profondeur de $r$ cette quantité $K(r)$ (voir au $§ 5.3$ ci-dessous la définition précise). On a alors $\alpha_{K}(r)=0$ et $\alpha_{k}(r)>0$ pour $0 \leqslant k<K$. On pose

$$
\Phi(r)=\sum_{0 \leqslant k<K} \alpha_{0}(r) \alpha_{1}(r) \cdots \alpha_{k-1}(r) \log \left(1 / \alpha_{k}(r)\right) .
$$

On a par exemple $\Phi(1 / k)=\log k$ si $k$ est entier, $k \geqslant 2$. Par convention, on pose $\Phi(0)=\Phi(1)=0$. L'équation fonctionnelle (2) est alors encore valable si $x \in] 0,1[\cap \mathbb{Q}$.

Théorème 2. Soit $x_{0} \in[0,1]$. On a

(i) $\Psi\left(x_{0}+h\right)-\Psi\left(x_{0}\right)=o(h \log (1 /|h|)) \quad\left(h \rightarrow 0, x_{0} \in X\right)$

(ii) $\frac{\Psi\left(x_{0}+h\right)-\Psi\left(x_{0}\right)}{h} \rightarrow \Phi\left(x_{0}\right) \quad\left(h \rightarrow 0, x_{0} \in X\right)$

(iii) $\Psi\left(x_{0}+h\right)-\Psi\left(x_{0}\right)$

$$
\begin{aligned}
& =\frac{1}{q} h \log (1 /|h|)+\left(\frac{1}{q}-2 \frac{\log q}{q}+\Phi\left(x_{0}\right)\right) h+O\left(q h^{2} \log \left(\left(q^{2}|h|\right)^{-1}\right)\right) \\
& \left(x_{0}=\frac{p}{q}, p \in \mathbb{N}, q \in \mathbb{N}^{*},(p, q)=1,|h|<2 / 3 q^{2}\right),
\end{aligned}
$$

où la constante implicite dans le $O$ est absolue.

Soit

$$
\omega(h)=\sup \{|\Psi(x)-\Psi(y)|, 0 \leqslant x, y \leqslant 1,|x-y| \leqslant h\}
$$

le module de continuité de la fonction $\Psi$. Notre troisième résultat fournit un équivalent asymptotique pour $\omega(h)$ quand $h$ tend vers 0 .

Théorème 3. On $a$

$$
\omega(h)=h \log (1 / h)+O(h) \quad(0<h \leqslant 1) .
$$

Nous emploierons désormais les notations

$$
\beta_{k}(x)=\alpha_{0}(x) \alpha_{1}(x) \cdots \alpha_{k}(x)
$$

(par convention, $\beta_{-1}=1$ ) et

$$
\gamma_{k}(x)=\beta_{k-1}(x) \log \left(1 / \alpha_{k}(x)\right) \quad(x \in X, k \geqslant 0),
$$


de sorte que $\gamma_{0}(x)=\log (1 / x)$ et que

$$
\begin{aligned}
\Phi(x) & =\sum_{k \geqslant 0} \gamma_{k}(x) \\
& =\sum_{k<K} \gamma_{k}(x)+\beta_{K-1}(x) \Phi\left(\alpha_{K}(x)\right) \quad(x \in X, K \in \mathbb{N}) .
\end{aligned}
$$

L'identité (8) est une forme généralisée de l'équation fonctionnelle (2).

Le $\S 2$ contient quelques remarques générales sur le comportement local moyen des fonctions localement intégrables. Au §3, nous rappelons les éléments de la théorie des fractions continues qui nous seront utiles. Nous obtenons au $\S 4$ une première majoration du module de continuité $\omega(h)$, essentiellement grâce au théorème de Ryll-Nardzewski. Pour la suite, notre approche est fondée sur un découpage diophantien de $X$ qui fait intervenir la notion de cellule, déjà sous-jacente dans [8]. Nous définissons cette notion et l'étudions en détail au $\S 5$, en introduisant notamment les notions de profondeur et d'épaisseur d'un sous-intervalle de ]0,1[. Nous évaluons ensuite l'intégrale des fonctions $\gamma_{k}$ sur un intervalle au $\S 6$. Au $\S 7$ nous démontrons le point (iii) du théorème 2 . Après avoir traité le cas des nombres de Cremer au $\S 8$ nous démontrons le théorème 1 au $\S 9$ et le théorème 3 au $\S 10$. Au $\S 11$ final, nous formulons des questions ouvertes.

Toutes les constantes implicites dans les symboles $O$ sont absolues. Cela étant, les calculs conduisent naturellement à des majorations numériquement explicites, et nous avons généralement adopté ce mode de présentation. Enfin, nous emploierons la notation d'Iverson : $[A]=1$ si la propriété $A$ est vérifiée, $[A]=0$ sinon.

\section{GÉnÉRAlités}

Soit $I$ un intervalle de $\mathbb{R}, X$ une partie de $I$ telle que $I \backslash X$ est de mesure nulle, et $f: X \rightarrow \mathbb{R}$ une fonction intégrable sur chaque segment inclus dans $I$. Pour fixer les idées, nous supposerons que $0 \in I$.

Considérons les quatre ensembles suivants :

- l'ensemble $\mathcal{C}$ des points de $X$ où $f$ est continue;

- l'ensemble $\mathcal{L}$ des points $x_{0} \in X$ tels que

$$
\frac{1}{h} \int_{x_{0}-h / 2}^{x_{0}+h / 2}\left|f(x)-f\left(x_{0}\right)\right| d x \rightarrow 0 \quad(h \rightarrow 0)
$$

- l'ensemble $\mathcal{L}^{1}$ des points $x_{0} \in I$ tels qu'il existe $\alpha \in \mathbb{R}$ avec

$$
\frac{1}{h} \int_{x_{0}-h / 2}^{x_{0}+h / 2}|f(x)-\alpha| d x \rightarrow 0 \quad(h \rightarrow 0) ;
$$

- l'ensemble $\mathcal{D}$ des points de dérivabilité de la fonction

$$
F(x)=\int_{0}^{x} f(t) d t \quad(x \in I) .
$$


L'ensemble $\mathcal{L}$ est l'ensemble de Lebesgue de $f$, et $\mathcal{L}^{1}$ est l' « ensemble de Lebesgue $L^{1} \gg$ de $f$ (voir par exemple [4], §2). On a

$$
\mathcal{C} \subset \mathcal{L} \subset \mathcal{L}^{1} \subset \mathcal{D}
$$

chaque inclusion pouvant être stricte. L'ensemble $I \backslash \mathcal{L}$ est de mesure nulle (théorème de Lebesgue).

Le cas de la fonction de Brjuno, que nous analysons en détail dans cet article, illustre la question générale suivante. Soit $f_{n}: I \rightarrow[0, \infty[(n \geqslant 0)$ une suite de fonctions intégrables telle que

$$
f(x)=\sum_{n \geqslant 0} f_{n}(x)
$$

soit intégrable sur $I$. Comment déterminer les ensembles $\mathcal{C}, \mathcal{L}, \mathcal{L}^{1}$ et $\mathcal{D}$ à partir de la connaissance des fonctions $f_{n}$ ?

Sans entrer plus avant dans l'étude de ce problème en toute généralité, contentons-nous d'indiquer un exemple montrant qu'un point $x_{0} \in I$ tel que :

- chaque fonction $f_{n}$ est continue en $x_{0}$

et

- la série $f\left(x_{0}\right)$ converge,

n'est pas nécessairement un point de dérivabilité de $F$. Il suffit de prendre $I=\mathbb{R}, f_{0}=0$ et, pour $n \geqslant 1$,

$$
f_{n}(t)=|t| \cdot\left[|t| \leqslant n^{-2 / 3}\right] .
$$

Chaque $f_{n}$ est continue en 0 avec $f_{n}(0)=0$, donc $f(0)=0$. Cependant $f(x)=|x|^{-1 / 2}-|x|\left\{|x|^{-3 / 2}\right\}(x \neq 0)$, donc $F$ n'est pas dérivable en 0.

Si $f$ est la fonction de Brjuno $\Phi$, on a $\mathcal{C}=\emptyset$ (la fonction de Brjuno est non bornée au voisinage de tout point de [0,1], au vu, par exemple, du point (iii) du théorème 2) et $\mathcal{L}=\mathcal{L}^{1}=\mathcal{D}$ est l'ensemble des nombres de Brjuno, d'après le théorème 1 et le point $(i i)$ du théorème 2 .

\section{Fractions CONTINUES}

Dans cette section nous rappelons quelques éléments de la théorie des fractions continues, et nous donnons des estimations élémentaires concernant les fonctions $\alpha_{k}$ et $\beta_{k}$.

3.1. Quotients incomplets, réduites. Pour $k \in \mathbb{N}$, nous définissons la fonction $^{\S}$

$$
a_{k}: X \rightarrow \mathbb{N}
$$

par les relations $a_{0}=0$ et $a_{k}=\left\lfloor 1 / \alpha_{k-1}\right\rfloor \quad(k \geqslant 1)$. Les fonctions $a_{k}, k \geqslant 1$, sont à valeurs dans $\mathbb{N}^{*}$. On appelle $a_{k}(x)$ le $《 k^{\mathrm{e}}$ quotient incomplet $»$ de $x$.

\footnotetext{
$\S$. Pour tout ce qui concerne ce paragraphe on pourra se référer par exemple à [11], chapter 7 .
} 
Avec la notation classique

$$
\left[X_{0} ; X_{1}, \ldots, X_{k}\right]=X_{0}+\frac{1}{X_{1}+\frac{1}{X_{2}+\frac{1}{\ddots+\frac{1}{X_{k-1}+\frac{1}{X_{k}}}}}}
$$

on a pour tout $k \geqslant 0$,

$$
x=\left[a_{0}(x) ; a_{1}(x), \ldots, a_{k-1}(x), a_{k}(x)+\alpha_{k}(x)\right] \quad(x \in X) .
$$

On définit aussi les fonctions $p_{k}, q_{k}: X \rightarrow \mathbb{N}$ par les relations

$$
\begin{aligned}
p_{-1} & =1 & q_{-1} & =0 \\
p_{0} & =0 & q_{0} & =1 \\
p_{1} & =1 & q_{1} & =a_{1} \\
\vdots & & \vdots & \\
p_{k} & =a_{k} p_{k-1}+p_{k-2} & q_{k} & =a_{k} q_{k-1}+q_{k-2} \quad(k \geqslant 1)
\end{aligned}
$$

de sorte que

$$
\left[a_{0} ; a_{1}, \ldots, a_{k-1}, t\right]=\frac{p_{k-1} t+p_{k-2}}{q_{k-1} t+q_{k-2}} \quad(k \geqslant 1, t>0),
$$

et

$$
\left[a_{0} ; a_{1}, \ldots, a_{k}\right]=\frac{p_{k}}{q_{k}} .
$$

Les fonctions $q_{k}(k \geqslant 0)$ et $p_{k}(k \geqslant 1)$ sont à valeurs dans $\mathbb{N}^{*}$. La fraction $p_{k}(x) / q_{k}(x)$ est appelée la « $k^{\mathrm{e}}$ réduite » de $x$. Elle est irréductible car

$$
p_{k} q_{k-1}-q_{k} p_{k-1}=(-1)^{k-1} \text {. }
$$

Une autre identité dont nous aurons l'usage est

$$
p_{k+1} q_{k-1}-p_{k-1} q_{k+1}=(-1)^{k-1} a_{k+1} .
$$

La suite $\left\{q_{k}\right\}_{k \geqslant-1}$ est à croissance au moins exponentielle ${ }^{\top}$ En effet, on a pour tout $k \geqslant 1$

$$
q_{k}=a_{k} q_{k-1}+q_{k-2} \geqslant q_{k-1}+q_{k-2} \geqslant 2 q_{k-2}
$$

En particulier,

$$
q_{k} \geqslant F_{k+1} \quad(k \geqslant 0),
$$

\footnotetext{
ๆ. Nous entendons par là l'existence d'une constante $c>1$ telle que $q_{k} \geqslant c^{k}$. Mais on n'a pas forcément $\liminf _{k \rightarrow \infty} q_{k+1} / q_{k}>1$.
} 
où $F_{n}$ est le $n^{\mathrm{e}}$ nombre de Fibonacci $\left(F_{0}=0, F_{1}=1\right.$ et $F_{n+1}=F_{n-1}+F_{n}$ pour $n \geqslant 1)$. On déduit également de (11) que

$$
\begin{aligned}
\sum_{j=0}^{k} q_{j} & =\sum_{j=2}^{k} q_{j-2}+q_{k-1}+q_{k} \\
& \leqslant \sum_{j=2}^{k}\left(q_{j}-q_{j-1}\right)+q_{k-1}+q_{k} \\
& =2 q_{k}+q_{k-1}-q_{1} \\
& \leqslant 3 q_{k},
\end{aligned}
$$

majoration valable pour tout $k \in \mathbb{N}$.

Nous utiliserons à plusieurs reprises une majoration de la somme des inverses des nombres de Fibonacci :

$$
\sum_{k \geqslant 0} 1 / F_{k+1} \leqslant 3,36
$$

3.2. Fonctions $\alpha_{k}$ et $\beta_{k}$. De la formule

$$
\begin{aligned}
x & =\left[a_{0}(x) ; a_{1}(x), \ldots, a_{k-1}(x), a_{k}(x)+\alpha_{k}(x)\right] \\
& =\frac{p_{k}(x)+\alpha_{k}(x) p_{k-1}(x)}{q_{k}(x)+\alpha_{k}(x) q_{k-1}(x)},
\end{aligned}
$$

on déduit

$$
\alpha_{k}(x)=-\frac{p_{k}(x)-x q_{k}(x)}{p_{k-1}(x)-x q_{k-1}(x)} .
$$

Cela implique les identités

$$
\begin{aligned}
\beta_{k}(x) & =(-1)^{k-1}\left(p_{k}(x)-x q_{k}(x)\right) \\
& =\frac{1}{q_{k+1}(x)+\alpha_{k+1}(x) q_{k}(x)} \quad(x \in X) .
\end{aligned}
$$

On dispose par conséquent de l'encadrement

$$
\frac{1}{q_{k+1}+q_{k}} \leqslant \beta_{k} \leqslant \frac{1}{q_{k+1}} \quad(k \geqslant-1) .
$$

Une majoration plus générale nous sera utile : la définition (5) entraîne

$$
\beta_{i+j}=\beta_{i} \cdot \beta_{j-1} \circ \alpha_{i+1} \quad(i \geqslant-1, j \geqslant 0),
$$

d'ou l'on déduit, d'après (19) et (12),

$$
\beta_{i+j} \leqslant \frac{1}{q_{i+1} F_{j+1}} .
$$

Par ailleurs, notant que $\left\lfloor 1 / \alpha_{k}\right\rfloor=a_{k+1}$ et que $a_{k+1}=\left(q_{k+1}-q_{k-1}\right) / q_{k}$, nous avons d'après (11)

$$
\frac{q_{k+1}}{2 q_{k}} \leqslant \frac{1}{\alpha_{k}} \leqslant a_{k+1}+1 \quad(k \geqslant 0) .
$$


3.3. Comparaison des fonctions $\gamma_{k}$ et $q_{k}^{-1} \log q_{k+1}$. Les fonctions $\gamma_{k}$ et $q_{k}^{-1} \log q_{k+1}$ sont proches l'une de l'autre. La proposition suivante exprime précisément ce fait.

Proposition 1. Pour $k \geqslant 0$, on a

$$
-\frac{\log \left(2 q_{k}\right)}{q_{k}} \leqslant \gamma_{k}-\frac{\log q_{k+1}}{q_{k}} \leqslant[k=0] \frac{\log 2}{q_{k}} .
$$

\section{Démonstration}

D'après (18), on a

$$
\frac{\log q_{k+1}}{q_{k}}-\gamma_{k}=\frac{q_{k} \log \left(q_{k+1} \alpha_{k}\right)+\alpha_{k} q_{k-1} \log q_{k+1}}{q_{k}\left(q_{k}+\alpha_{k} q_{k-1}\right.} \geqslant \frac{q_{k} \log \left(q_{k+1} \alpha_{k}\right)}{q_{k}\left(q_{k}+\alpha_{k} q_{k-1}\right)} .
$$

Or pour $k>0, q_{k-1} \geqslant 1$, et donc

$$
q_{k+1} \alpha_{k}=\frac{a_{k+1} q_{k}+q_{k-1}}{a_{k+1}+\alpha_{k+1}} \geqslant 1,
$$

On obtient ainsi la deuxième inégalité de (23) lorsque $k>0$, c'est-é-dire,

$$
\gamma_{k} \leqslant \frac{\log q_{k+1}}{q_{k}}
$$

Pour $k=0$, on a

$$
\gamma_{0}-\frac{\log q_{1}}{q_{0}}=\log (1 / x)-\log a_{1}=\log \left(\frac{a_{1}+\alpha_{1}}{a_{1}}\right) \leqslant \log \left(\frac{a_{1}+1}{a_{1}}\right) \leqslant \log 2 .
$$

Par ailleurs,

$$
\gamma_{k}=\frac{\log \left(1 / \alpha_{k}\right)}{q_{k}+\alpha_{k} q_{k-1}}
$$

est une fonction décroissante de $\alpha_{k}$. Comme $\alpha_{k} \leqslant 1 / a_{k+1}$, on a donc

$$
\gamma_{k} \geqslant \frac{\log a_{k+1}}{q_{k}+q_{k-1} / a_{k+1}}=\frac{a_{k+1} \log a_{k+1}}{q_{k+1}} .
$$

Pour montrer que

il suffit donc de montrer que

$$
\gamma_{k}-\frac{\log q_{k+1}}{q_{k}} \geqslant-\frac{\log \left(2 q_{k}\right)}{q_{k}},
$$

$$
a_{k+1} \log a_{k+1} \geqslant \frac{q_{k+1}}{q_{k}} \log \frac{q_{k+1}}{2 q_{k}} .
$$

Si $a_{k+1}=1$, on a $q_{k+1}=q_{k}+q_{k-1} \leqslant 2 q_{k}$, donc (24) est vérifièe. Si $a_{k+1} \geqslant 2$, on a $\frac{q_{k+1}}{q_{k}} \geqslant 2$ et

$$
a_{k+1}=\frac{q_{k+1}-q_{k-1}}{q_{k}} \geqslant \frac{q_{k+1}}{q_{k}}-1 .
$$

L'inégalité (24) résultera alors de

$$
(x-1) \log (x-1) \geqslant x \log (x / 2) \quad(x \geqslant 2)
$$

ce qui découle de l'étude du sens de variation de la différence

$$
(x-1) \log (x-1)-x \log (x / 2)
$$

$\operatorname{sur}[2, \infty[$. 
En particulier, $x \in X$ est un nombre de Brjuno si et seulement si la série

$$
\sum_{k \geqslant 0} \frac{\log q_{k+1}(x)}{q_{k}(x)}
$$

est convergente.

\section{Majoration du module de COntinuité de $\Psi$}

Sans utiliser la notion de cellule (§5), on peut déjà donner une majoration utile du module de continuité de $\Psi$, proposition 3 ci-dessous. Pour la démontrer, nous majorons d'abord l'intégrale de $\log \left(1 / \alpha_{k}\right)$ sur un intervalle de longueur $h$, proposition 2 ci-dessous.

Notre démonstration de la proposition 2 requiert une majoration de la fonction $\Gamma$ d'Euler, sans doute classique mais pour laquelle nous ne disposons pas de référence. Rappelons que pour $x>0$,

$$
\Gamma(x)=\int_{0}^{\infty} e^{-t} t^{x-1} d t=\int_{0}^{1}(\log 1 / t)^{x-1} d t .
$$

Lemme 1. Pour $q \in[2, \infty[$, on a

$$
2 \Gamma(q+1) \leqslant q^{q} .
$$

\section{Démonstration}

Notons $\psi$ la dérivée logarithmique de la fonction $\Gamma$. L'inégalité proposée est vraie pour $q=2$. Ensuite, il suffit de voir que les dérivées logarithmiques des deux membres vérifient la méme inégalité pour $q \geqslant 2$, c'est-é-dire que

$$
\psi(q+1) \leqslant \log q+1 \quad(q \geqslant 2) .
$$

Or, nous disposons de la formule classique

$$
\psi(x)=-\gamma-\frac{1}{x}+\sum_{n \geqslant 1} \frac{1}{n}-\frac{1}{n+x} \quad(x \neq 0,-1,-2, \ldots),
$$

où $\gamma$ désigne la constante d'Euler. Pour $x>0$, on a donc

$$
\begin{aligned}
\psi(x) & =-\gamma-\frac{1}{x}+\sum_{n \geqslant 1} \frac{x}{n(n+x)} \\
& \leqslant-\gamma-\frac{1}{x}+1-\frac{1}{x+1}+\int_{1}^{\infty}\left(\frac{1}{t}-\frac{1}{t+x}\right) d t \\
& =1-\gamma-\frac{1}{x}-\frac{1}{x+1}+\log (x+1) .
\end{aligned}
$$

Par conséquent,

$$
\begin{aligned}
\psi(q+1)-\log q & \leqslant \log \left(1+\frac{2}{q}\right)-\frac{1}{q+1}-\frac{1}{q+2}+1-\gamma \\
& \leqslant 1 \quad(q \geqslant 2) .
\end{aligned}
$$

Proposition 2. Soit I un intervalle de longueur $h \leqslant e^{-2}$, inclus dans $[0,1]$.

On a pour tout $k \in \mathbb{N}$

$$
\int_{I} \log \left(1 / \alpha_{k}(x)\right) d x \leqslant e h \log (1 / h)
$$




\section{Démonstration}

Pour $q>1$ et $p=q /(q-1)$, l'inégalité de Hölder donne

$$
\int_{I} \log \left(1 / \alpha_{k}(x)\right) d x \leqslant h^{1 / p}\left(\int_{0}^{1} \log ^{q}\left(1 / \alpha_{k}(x)\right) d x\right)^{1 / q} .
$$

Comme $\alpha$ laisse la mesure $\mu$ invariante, nous avons

$$
\begin{aligned}
\int_{0}^{1} \log ^{q}\left(1 / \alpha_{k}(x)\right) d x & \leqslant 2 \int_{0}^{1} \log ^{q}\left(1 / \alpha_{k}(x)\right) \frac{d x}{1+x}=2 \int_{0}^{1} \log ^{q}(1 / x) \frac{d x}{1+x} \\
& \leqslant 2 \int_{0}^{1} \log ^{q}(1 / x) d x=2 \Gamma(q+1) .
\end{aligned}
$$

D'après le lemme 1 , il suit

$$
\int_{I} \log \left(1 / \alpha_{k}(x)\right) d x \leqslant q h^{1-1 / q} \quad(q \geqslant 2) .
$$

En effectuant le choix $q=\log (1 / h)$, pour lequel $q \geqslant 2$ d'après l'hypothése faite sur $h$, nous obtenons la majoration annoncée.

Proposition 3. On a

$$
\omega(h) \leqslant 10 h \log (1 / h) \quad\left(0<h \leqslant e^{-2}\right) .
$$

\section{Démonstration}

On a pour $0<h \leqslant e^{-2}$,

$$
\begin{aligned}
\Psi(x+h)-\Psi(x) & =\int_{x}^{x+h} \Phi(t) d t=\sum_{k \geq 0} \int_{x}^{x+h} \gamma_{k}(t) d t \\
& \leqslant \sum_{k \geq 0} \frac{1}{F_{k+1}} \int_{x}^{x+h} \log \left(1 / \alpha_{k}(t)\right) d t \quad \text { (d'après (19) puis (12)) } \\
& \leqslant e h \log (1 / h) \sum_{k \geq 0} \frac{1}{F_{k+1}} \quad \text { (d'après la proposition 2) } \\
& \leqslant 3,36 e h \log (1 / h) \quad(\text { d'après (14)) }
\end{aligned}
$$

et cela entraîne bien la majoration annoncée.

\section{Notion De CELlule}

5.1. Définition et propriètés. Soit $b_{0}=0, b_{1}, \ldots, b_{k} \in \mathbb{N}^{*}$. La cellule (de profondeur $k) \mathfrak{c}\left(b_{1}, \ldots, b_{k}\right)$ est l'intervalle ouvert d'extrémités $\left[b_{0} ; b_{1}, \ldots, b_{k}\right]$ et $\left[b_{0} ; b_{1}, \ldots, b_{k-1}, b_{k}+1\right]$. Par convention, la seule cellule de profondeur 0 est $] 0,1[$.

La notion de cellule n'est pas nouvelle : elle intervient naturellement dans le cadre de la théorie métrique et ergodique des fractions continues sous l'appelation de cylindre (cf [2] page 30 et chapitre 3.5) ou d'intervalle (fondamental) d'ordre $n$ (cf [6] §12 ou [1] §4 par exemple).

Nous énonçons à présent quelques propriétés élémentaires concernant les cellules. 
- Dans la cellule $\mathfrak{c}\left(b_{1}, \ldots, b_{k}\right)$, les fonctions $a_{j}, p_{j}, q_{j}$ sont constantes pour $j \leqslant k$ :

$$
a_{j}(x)=b_{j}, \quad \frac{p_{j}(x)}{q_{j}(x)}=\left[b_{0} ; b_{1}, \ldots, b_{j}\right] \quad\left(x \in \mathfrak{c}\left(b_{1}, \ldots, b_{j}\right)\right) .
$$

- La cellule $\mathfrak{c}\left(b_{1}, \ldots, b_{k}\right)$ est l'intervalle ouvert d'extrémités

$$
\frac{p_{k}}{q_{k}} \quad \text { et } \quad \frac{p_{k}+p_{k-1}}{q_{k}+q_{k-1}}
$$

(dans cet ordre si $k$ est pair ; dans l'ordre opposé si $k$ est impair). Sa longueur est

$$
\frac{1}{q_{k}\left(q_{k}+q_{k-1}\right)}
$$

- On a

$$
\alpha\left(\mathfrak{c}\left(b_{1}, \ldots, b_{k}\right) \cap X\right)=\mathfrak{c}\left(b_{2}, \ldots, b_{k}\right) \cap X .
$$

- Soit $k \geqslant 1$, et $\mathfrak{c}=\mathfrak{c}\left(b_{1} \ldots, b_{k}\right)$ une cellule de profondeur $k$. Les cellules de profondeur $k+1$ incluses dans $\mathfrak{c}$ forment une suite $\left(\mathfrak{c}_{n}\right)_{n \geqslant 1}$ d'intervalles ouverts qui constitue une partition de $\mathfrak{c}$ privée d'une partie de $\mathbb{Q}$ (les rationnels de la forme $\left[b_{0} ; b_{1}, \ldots, b_{k}, n\right], n$ décrivant $\left.\mathbb{N}^{*}\right)$.

La cellule $\mathfrak{c}_{n}$ est l'intervalle d'extrémités

$$
\begin{aligned}
{\left[b_{0} ; b_{1}, \ldots, b_{k}, n\right] } & =\frac{n p_{k}+p_{k-1}}{n q_{k}+q_{k-1}} \\
\text { et }\left[b_{0} ; b_{1}, \ldots, b_{k-1}, b_{k}, n+1\right] & =\frac{(n+1) p_{k}+p_{k-1}}{(n+1) q_{k}+q_{k-1}} .
\end{aligned}
$$

Ainsi $\mathfrak{c}_{n}$ et $\mathfrak{c}_{n+1}$ sont contigües, et $\mathfrak{c}_{n}$ est l'ensemble des nombres de la forme

$$
\frac{s p_{k}+p_{k-1}}{s q_{k}+q_{k-1}}, \quad n<s<n+1
$$

Dans $\mathfrak{c}_{n}$, la fonction $q_{k+1}$ a la valeur constante $n q_{k}+q_{k-1}$. Observons que $\frac{p_{k}+p_{k-1}}{q_{k}+q_{k-1}}$ est l'une des extrémités de $\mathfrak{c}_{1}$, mais que $\frac{p_{k}}{q_{k}}$ n'est l'extrémité d'aucune cellule $\mathfrak{c}_{n}$. En revanche, $\frac{p_{k}}{q_{k}}$ est la limite quand $n$ tend vers l'infini des extrémités de $\mathfrak{c}_{n}$.

5.2. Distance d'un irrationnel donné au bord de la cellule de profondeur $k$ qui le contient. Soit $x \in X$ et $k \in \mathbb{N}$. Il existe une unique cellule de profondeur $k$ qui contient $x$ :

$$
\mathfrak{c}=\left\{\begin{array}{l}
] \frac{p_{k}}{q_{k}}, \frac{p_{k}+p_{k-1}}{q_{k}+q_{k-1}}[\text { si } k \text { est pair } \\
] \frac{p_{k}+p_{k-1}}{q_{k}+q_{k-1}}, \frac{p_{k}}{q_{k}}[\text { si } k \text { est impair }
\end{array}\right.
$$

où l'on a posé $p_{j}=p_{j}(x), q_{j}=q_{j}(x)$ pour $j \geqslant-1$. La distance de $x$ au bord de $\mathfrak{c}$ joue un rôle déterminant dans la suite de notre travail. Nous la notons $\delta_{k}(x)$. On a par exemple

$$
\delta_{0}(x)=\min (x, 1-x) \quad(x \in X) .
$$


Observons tout de suite que la suite $k \mapsto \delta_{k}(x)$ est décroissante (au sens large) et tend vers 0 quand $k$ tend vers l'infini. Cherchons maintenant à exprimer $\delta_{k}(x)$ en termes des fonctions précédemment définies. On a d'abord, d'après (17),

$$
x-\frac{p_{k}}{q_{k}}=(-1)^{k} \frac{\beta_{k}(x)}{q_{k}} .
$$

Pour la distance de $x$ à l'autre extrémité de $\mathfrak{c}$, on a

$$
\begin{aligned}
x-\frac{p_{k}+p_{k-1}}{q_{k}+q_{k-1}} & =(-1)^{k-1} \frac{\beta_{k+1}(x)}{q_{k+1}}+\left(\frac{p_{k+1}}{q_{k+1}}-\frac{p_{k}+p_{k-1}}{q_{k}+q_{k-1}}\right) \\
& =(-1)^{k-1}\left(\frac{\beta_{k+1}(x)}{q_{k+1}}+\frac{a_{k+1}-1}{q_{k+1}\left(q_{k}+q_{k-1}\right)}\right),
\end{aligned}
$$

où la deuxième égalité résulte des identités (9) et (10). Nous obtenons ainsi la formule

$$
\delta_{k}=\min \left(\frac{\beta_{k}}{q_{k}}, \frac{\beta_{k+1}}{q_{k+1}}+\frac{a_{k+1}-1}{q_{k+1}\left(q_{k}+q_{k-1}\right)}\right) .
$$

Proposition 4. Pour $k \in \mathbb{N}$ on a

$$
\delta_{k} \leqslant \frac{1}{q_{k} q_{k+1}} \quad \text { et } \quad \delta_{k} \geqslant \begin{cases}\frac{1 / 2}{q_{k+1} q_{k+2}} & \text { si } a_{k+1}=1 \\ \frac{1 / 2}{q_{k} q_{k+1}} & \text { si } a_{k+1} \geqslant 2 .\end{cases}
$$

\section{Démonstration}

On a d'abord, d'après (19),

$$
\delta_{k} \leqslant \frac{\beta_{k}}{q_{k}} \leqslant \frac{1}{q_{k} q_{k+1}} .
$$

Ensuite, si $a_{k+1}=1$, toujours d'après (19),

$$
\delta_{k}=\frac{\beta_{k+1}}{q_{k+1}} \geqslant \frac{1 / 2}{q_{k+1} q_{k+2}},
$$

et si $a_{k+1} \geqslant 2$,

$$
\delta_{k} \geqslant \min \left(\frac{1}{q_{k}\left(q_{k}+q_{k+1}\right)}, \frac{1}{q_{k+1}\left(q_{k}+q_{k-1}\right)}\right) \geqslant \frac{1 / 2}{q_{k} q_{k+1}} .
$$

La proposition suivante sera utilisée au $\S 6.3$ lors de la preuve de la proposition 11 .

Proposition 5. Pour $k \in \mathbb{N}$ on a

$$
\frac{\log \left(\left(2 \delta_{k}\right)^{-1}\right)}{q_{k}} \leqslant 2 \frac{\log q_{k+1}}{q_{k}}+2 \frac{\log q_{k+2}}{q_{k+1}} .
$$

\section{Démonstration}

Si $a_{k+1} \geqslant 2$ on a, d'après la proposition 4 ,

$$
\frac{\log \left(\left(2 \delta_{k}\right)^{-1}\right)}{q_{k}} \leqslant \frac{\log \left(q_{k} q_{k+1}\right)}{q_{k}} \leqslant 2 \frac{\log q_{k+1}}{q_{k}} .
$$


Si $a_{k+1}=1$, on a

$$
\frac{\log \left(\left(2 \delta_{k}\right)^{-1}\right)}{q_{k}} \leqslant \frac{\log \left(q_{k+1} q_{k+2}\right)}{q_{k}} .
$$

Mais dans ce cas on a aussi $q_{k+1} \leqslant 2 q_{k}$, d'où

$$
\frac{\log q_{k+2}}{q_{k}} \leqslant 2 \frac{\log q_{k+2}}{q_{k+1}}
$$

ce qui démontre l'inégalité annoncée.

5.3. Profondeur d'un nombre rationnel. Soit $r$ un nombre rationnel, $0<r<1$, mis sous forme irréductible $p / q$. Il peut s'écrire d'une et une seule faîon sous la forme

$$
r=\left[0 ; b_{1}, \ldots, b_{k}\right]
$$

avec $k \in \mathbb{N}^{*}, b_{i} \in \mathbb{N}^{*}, 1 \leqslant i \leqslant k$, et $b_{k} \geqslant 2$. Nous dirons alors que $r$ est de profondeur $\|^{k} k$. Par convention, 0 et 1 sont de profondeur 0 .

Écrivons $\left[0 ; b_{1}, \ldots, b_{k-1}\right]$ sous forme réduite $p_{k-1} / q_{k-1}$ (si $k=1$, on a $p_{0}=0$ et $\left.q_{0}=1\right)$. Le nombre rationnel $r$ est une extrémité de deux cellules de profondeur $k$ (qui sont donc contigées) :

$$
\begin{aligned}
& \mathfrak{c} \text { d'extrémités }\left[0 ; b_{1}, \ldots, b_{k-1}, b_{k}-1\right]=\frac{p-p_{k-1}}{q-q_{k-1}} \text { et } r ; \\
& \mathfrak{c}^{\prime} \text { d'extrémités } r \text { et }\left[0 ; b_{1}, \ldots, b_{k-1}, b_{k}+1\right]=\frac{p+p_{k-1}}{q+q_{k-1}} .
\end{aligned}
$$

Comme $b_{k} \geqslant 2$, on a $q_{k-1} \leqslant q / 2$. La longueur de $\mathfrak{c}$ est

$$
\frac{1}{\left(q-q_{k-1}\right) q}>\frac{1}{q^{2}}
$$

tandis que la longueur de $\mathfrak{c}^{\prime}$ est

$$
\frac{1}{q\left(q+q_{k-1}\right)} \geqslant \frac{2}{3 q^{2}}
$$

En particulier, on a l'inclusion

$$
] r-\frac{2}{3 q^{2}}, r+\frac{2}{3 q^{2}}\left[\backslash\{r\} \subset \mathfrak{c} \cup \mathfrak{c}^{\prime} .\right.
$$

5.4. Profondeur et épaisseur d'un segment inclus dans ]0,1[. Soit $I=[a, b]$ un segment inclus dans $] 0,1[$, de longueur $h=b-a>0$ et d'extrémités $a, b$ irrationnelles. Il existe un unique entier naturel $K$ tel que $I$ soit inclus dans une cellule de profondeur $K$, mais dans aucune cellule de profondeur $K+1$. Nous dirons alors que $I$ est de profondeur $K$. Dans le cas où $x=(a+b) / 2$ est aussi irrationnel, le nombre $K$ est défini par l'encadrement

$$
\delta_{K+1}(x)<h / 2<\delta_{K}(x) .
$$

\footnotetext{
\|. On trouvera dans [10] une liste de problèmes arithmétiques sur la profondeur des nombres rationnels.
} 
Signalons une petite subtilité. Il est exact que la longueur d'un segment $I$ tend uniformément vers 0 quand sa profondeur $K$ tend vers l'infini : on a d'après (28) et (12)

$$
|I| \leqslant \frac{1}{F_{K+1} F_{K+2}}
$$

En revanche, $|I|$ peut tendre vers 0 alors que $K$ garde une valeur constante, 0 par exemple : il suffit que $1 / 2$ appartienne à $I$. Cela étant, dans le cas où $x \in X$ est fixé et $I=[x-h / 2, x+h / 2]$ avec $x \pm h / 2 \in X$, on a

$$
\lim _{h \rightarrow 0} K(x, h)=\infty
$$

puisque

$$
\frac{1 / 2}{q_{K+2}(x) q_{K+3}(x)}<h
$$

d'après (30) et la proposition 4.

Nous définissons également l'épaisseur de $I$ comme le nombre de cellules de profondeur $K+1$ qui ont une intersection non vide avec $I$, où $K$ est la profondeur de $I$. L'épaisseur de $I$ est un nombre entier supérieur ou égal à 2 .

5.5. Convention. Nous redéfinissons maintenant, et pour toute la suite, les fonctions $a_{k}, p_{k}, q_{k}, \alpha_{k}, \beta_{k}$ en les prolongeant par continuité (sans changer de notation) sur chaque cellule de profondeur $k$ (et donc également sur chaque cellule de profondeur supérieure à $k)$. Ainsi, en posant $\mathfrak{c}=\mathfrak{c}\left(b_{1}, \ldots, b_{k}\right)$, on aura pour $x \in \mathfrak{c}$ :

$$
\begin{aligned}
& a_{k}(x)=b_{k} \\
& \frac{p_{k}(x)}{q_{k}(x)}=\frac{p_{k}}{q_{k}}=\left[0 ; b_{1}, \ldots, b_{k}\right] \\
& \alpha_{k}(x)=\frac{q_{k} x-p_{k}}{-q_{k-1} x+p_{k-1}} \\
& \beta_{k}(x)=(-1)^{k-1}\left(p_{k}-x q_{k}\right) .
\end{aligned}
$$

En particulier, $\alpha_{k}$ est dérivable sur $\mathfrak{c}$ et y vérifie

$$
\alpha_{k}^{\prime}=(-1)^{k} \beta_{k-1}^{-2}=(-1)^{k}\left(q_{k}+\alpha_{k} q_{k-1}\right)^{2} .
$$

On a aussi la relation valable sur $\mathfrak{c}$

$$
\beta_{k-1}=\frac{1}{q_{k}}\left(1-q_{k-1} \beta_{k}\right) .
$$

\section{Estimations EN MOYEnNE DE $\gamma_{k}$ SUR Un INTERVAlle INCLUS DANS UNE CELLULE DE PROFONDEUR $K$}

Soit $I$ un intervalle inclus dans une cellule de profondeur $K$. Notre objectif est ici de majorer l'intégrale $\int_{I} \gamma_{k}(t) d t$, où $\gamma_{k}$ est définie par (6). Pour $j \leqslant K$, nous noterons $p_{j}, q_{j}$ les valeurs constantes de ces fonctions sur $I$. 
La discussion portera esssentiellement sur les tailles respectives de $k$ et $K$. Nous ferons fréquemment usage, sans toujours le mentionner explicitement, des encadrements (19) et (22). Enfin nous supposerons toujours que la longueur $h$ de $I$ vérifie $h \leqslant e^{-2}$.

6.1. Le cas $k<K$.

Proposition 6. Pour $k<K$ on a

$$
\begin{aligned}
\int_{I} \gamma_{k}(t) d t & \leqslant h \frac{\log \left(2 q_{k+1}\right)}{q_{k}} \\
& \leqslant \frac{h \log (1 / h)}{2 q_{k}}+\frac{h}{q_{k}} .
\end{aligned}
$$

\section{Démonstration}

La première inégalité résulte de (23). Pour la seconde, on observe que $I$ est inclus dans une cellule de profondeur $k+1$ dont la longueur est

$$
\frac{1}{q_{k+1}\left(q_{k+1}+q_{k}\right)}<\frac{1}{q_{k+1}^{2}}
$$

donc $q_{k+1}<h^{-1 / 2}$ et

$$
\begin{aligned}
\log \left(2 q_{k+1}\right) & \leqslant \log \left(h^{-1 / 2}\right)+\log 2 \\
& \leqslant \frac{1}{2} \log (1 / h)+1
\end{aligned}
$$

d'où le résultat.

Par ailleurs, lorsque $k<K$, nous disposons d'une majoration uniforme de la dérivée de $\gamma_{k}$ dans une cellule de profondeur $K$, ce qui nous conduit au résultat suivant.

Proposition 7. Soit I un segment de longueur $h$ inclus dans une cellule $\mathfrak{c}$ de profondeur $K$ et $x$ un point quelconque de $I$. Pour $k<K$, on a

$$
\int_{I}\left|\gamma_{k}(t)-\gamma_{k}(x)\right| d t \leqslant \frac{3}{2} q_{k+1} h^{2}
$$

\section{Démonstration}

D'après les formules (32) et (33), la fonction $\gamma_{k}$ est dérivable sur $\mathfrak{c}$ pour $k \leqslant K$, et (33) et (34) fournissent

$$
\gamma_{k}^{\prime}=(-1)^{k-1} q_{k-1} \log \left(1 / \alpha_{k}\right)+\frac{(-1)^{k-1}}{\beta_{k}} .
$$

Nous en déduisons, pour $k<K, t \in I$,

$$
\left|\gamma_{k}^{\prime}(t)\right| \leqslant q_{k-1} \log \left(a_{k+1}+1\right)+q_{k+1}+q_{k} \leqslant q_{k} a_{k+1}+q_{k-1}+2 q_{k+1}=3 q_{k+1} .
$$

La conclusion découle alors de l'inégalité des accroissements finis et de l'inégalité

$$
\int_{I}|t-x| d t \leqslant \frac{h^{2}}{2}
$$


6.2. Le cas $k=K$. Dans ce paragraphe et le suivant, nous donnons deux estimations, l'une comparant $\int_{I} \gamma_{K}(t) d t$ à $h \log 1 / h$, l'autre à $h$. Pour simplifier les formulations et les démonstrations qui suivent, nous supposons dans ces $\S \S 6.2$ et 6.3 que $I$ est un segment dont les extrémités et le milieu sont irrationnels. Nous noterons respectivement $h, x, K$ et $E$ la longueur, le milieu, la profondeur et l'épaisseur de $I$.

Pour la première estimation, un lemme élémentaire sera utile.

Lemme 2. On a

$$
\int_{I} \log (1 / t) d t \leqslant h \log (1 / h)+h
$$

\section{Démonstration}

Comme $t \mapsto \log (1 / t)$ est positive et décroissante sur $] 0,1]$, on a

$$
\begin{aligned}
\int_{I} \log (1 / t) d t & \leqslant \int_{0}^{h} \log (1 / t) d t \\
& =h \log (1 / h)+h .
\end{aligned}
$$

Proposition 8. On a

$$
\int_{I} \gamma_{K}(t) d t \leqslant \frac{h \log (1 / h)}{q_{K}}([E>2]+[E=2] / 2)+\frac{2 h}{q_{K}} .
$$

\section{Démonstration}

En effet,

$$
\begin{aligned}
\int_{I} \gamma_{K}(t) d t & =\int_{I} \beta_{K-1}(t) \log \beta_{K-1}(t) d t+\int_{I} \beta_{K-1}(t) \log 1 / \beta_{K}(t) d t \\
& \leqslant \int_{I} \beta_{K-1}(t) \log 1 / \beta_{K}(t) d t \quad\left(\operatorname{car} \beta_{K-1}(t) \leqslant 1\right) \\
& =\frac{1}{q_{K}^{2}} \int_{\beta_{K}(I)}\left(1-u q_{K-1}\right) \log (1 / u) d u
\end{aligned}
$$

où l'on a posé $u=\beta_{K}(t)$ et utilisé (35). On a donc

$$
\begin{aligned}
\int_{I} \gamma_{K}(t) d t & \leqslant \frac{1}{q_{K}^{2}} \int_{\beta_{K}(I)} \log (1 / u) d u \\
& \leqslant \frac{1}{q_{K}^{2}}\left|\beta_{K}(I)\right| \log 1 /\left|\beta_{K}(I)\right|+\frac{\left|\beta_{K}(I)\right|}{q_{K}^{2}} \quad \text { (d'après le lemme 2) } \\
& =\frac{1}{q_{K}^{2}}\left(q_{K} h\right) \log \left(\left(q_{K} h\right)^{-1}\right)+\frac{q_{K} h}{q_{K}^{2}} \\
& =\frac{h}{q_{K}} \log (1 / h)+\frac{\left(1-\log q_{K}\right) h}{q_{K}},
\end{aligned}
$$

ce qui démontre la proposition dans le cas où $E>2$.

Par ailleurs, si $E=2$, les nombres $x \pm h / 2$ se situent chacun dans l'une de deux cellules adjacentes, disons $\mathfrak{c}\left(b_{1}, \ldots, b_{K}, b_{K+1}-1\right)$ et $\mathfrak{c}\left(b_{1}, \ldots, b_{K}, b_{K+1}\right)$, où $b_{K+1} \geqslant 2$. Posons

$$
\left[0 ; b_{1}, \ldots, b_{K}, b_{K+1}\right]=\frac{p}{q} \quad((p, q)=1)
$$


et $q$ est d'ailleurs la valeur maximale de la fonction $q_{K+1}$ sur $I$.

La longueur de $I$ est inférieure à la somme des longueurs de ces deux cellules :

$$
h \leqslant\left|\frac{p+p_{K}}{q+q_{K}}-\frac{p-p_{K}}{q-q_{K}}\right|=\frac{2}{\left(q+q_{K}\right)\left(q-q_{K}\right)} .
$$

Comme $b_{K+1} \geqslant 2$, on a $q=b_{K+1} q_{K}+q_{K-1} \geqslant 2 q_{K}$, ce qui implique

$$
h \leqslant \frac{8 / 3}{q^{2}}
$$

et donc

$$
\log (2 q) \leqslant \frac{1}{2} \log (1 / h)+\log (2 \sqrt{8 / 3}) \leqslant \frac{1}{2} \log (1 / h)+2 .
$$

Enfin, nous avons

$$
\begin{aligned}
\int_{I} \gamma_{K}(t) d t & \leqslant \frac{1}{q_{K}} \int_{I} \log \left(2 q_{K+1}(t)\right) d t \quad \text { (d'après (23)) } \\
& \leqslant \frac{h}{q_{K}} \log (2 q) \\
& \leqslant \frac{h}{2 q_{K}} \log (1 / h)+\frac{2 h}{q_{K}} .
\end{aligned}
$$

Proposition 9. On a

$$
\int_{I} \gamma_{K}(t) d t \leqslant 8 h \gamma_{K}(x)+\frac{h}{q_{K}}\left(6 \log q_{K}+4\right) .
$$

\section{Démonstration}

D'après (19), on a

$$
\begin{aligned}
\int_{I} \gamma_{K}(t) d t & =\int_{I} \beta_{K-1}(t) \log \left(1 / \alpha_{K}(t)\right) d t \\
& =\int_{I} \beta_{K-1}(t)^{3} \cdot \beta_{K-1}(t)^{-2} \log \left(1 / \alpha_{K}(t)\right) d t \\
& \leqslant q_{K}^{-3} \int_{I} \beta_{K-1}(t)^{-2} \log \left(1 / \alpha_{K}(t)\right) d t .
\end{aligned}
$$

Comme $\alpha_{K}^{\prime}(t)=(-1)^{K} / \beta_{K-1}^{2}(t)$, il suit

$$
\int_{I} \gamma_{K}(t) d t \leqslant q_{K}^{-3} \int_{\alpha_{K}(I)} \log (1 / u) d u
$$

L'intervalle $\alpha_{K}(I)$ a pour extrémités $\alpha_{K}(x-h / 2)$ et $\alpha_{K}(x+h / 2)$. Or, d'après l'inégalité des accroissements finis,

$$
\left|\alpha_{K}(x \pm h / 2)-\alpha_{K}(x)\right| \leqslant(h / 2) \max _{\xi \in I} \beta_{K-1}(\xi)^{-2} \leqslant 2 q_{K}^{2} h .
$$

Supposons d'abord $4 q_{K}^{2} h \leqslant \alpha_{K}(x)$. Nous en déduisons

$$
\frac{1}{u} \leqslant \frac{2}{\alpha_{K}(x)} \quad\left(u \in \alpha_{K}(I)\right),
$$


donc,

$$
\begin{aligned}
\int_{I} \gamma_{K}(t) d t & \leqslant q_{K}^{-3} \log \left(2 / \alpha_{K}(x)\right) \int_{\alpha_{K}(I)} d u \leqslant q_{K}^{-3} \log \left(2 / \alpha_{K}(x)\right) \cdot 4 q_{K}^{2} h \\
& \leqslant 8 h \beta_{K-1}(x) \log \left(1 / \alpha_{K}(x)\right)+\frac{4 h}{q_{K}} \log 2 \\
& \leqslant 8 h \gamma_{K}(x)+\frac{3 h}{q_{K}} .
\end{aligned}
$$

Supposons ensuite $4 q_{K}^{2} h \geqslant \alpha_{K}(x)$. Nous avons dans ce cas, d'après la proposition 2,

$$
\begin{aligned}
\int_{I} \gamma_{K}(t) d t & \leqslant \max _{t \in I}\left|\beta_{K-1}(t)\right| \text { eh } \log (1 / h) \leqslant \frac{e}{q_{K}} h \log \left(4 q_{K}^{2} / \alpha_{K}(x)\right) \\
& =\frac{e}{q_{K}} h \log \left(1 / \alpha_{K}(x)\right)+\frac{h}{q_{K}}\left(2 e \log 2+2 e \log q_{K}\right) \\
& \leqslant 6 h \gamma_{K}(x)+\frac{h}{q_{K}}\left(6 \log q_{K}+4\right),
\end{aligned}
$$

d'où la conclusion.

6.3. Le cas $k>K$. Nos calculs nécessiteront une majoration élémentaire.

Lemme 3. Soit $m$ et $n$ des nombres entiers tels que $1 \leqslant m<n$. On a alors

$$
\sum_{m \leqslant \ell \leqslant n} \frac{1}{\ell^{3}} \leqslant 3 \frac{n-m}{m^{2} n} .
$$

\section{Démonstration}

On a

$$
\begin{aligned}
\sum_{m \leqslant \ell \leqslant n} \frac{1}{\ell^{3}} & \leqslant \frac{1}{m^{3}}+\int_{m}^{n} \frac{d t}{t^{3}}=\frac{1}{m^{3}}+\frac{1}{2}\left(\frac{1}{m^{2}}-\frac{1}{n^{2}}\right) \\
& =\frac{1}{m^{3}}+\frac{n^{2}-m^{2}}{2 m^{2} n^{2}} \leqslant \frac{1}{m^{3}}+\frac{n-m}{m^{2} n} .
\end{aligned}
$$

Maintenant, comme $1 \leqslant m \leqslant n-1$, on a $m(n-m) \geqslant n-1 \geqslant n / 2$, donc

d'où le résultat.

$$
\frac{1}{m^{3}} \leqslant 2 \frac{n-m}{m^{2} n}
$$

Nous sommes maintenant en mesure de traiter le cas $k>K$.

Proposition 10. Pour $k \in \mathbb{N}$ tel que $k>K$ on $a$

$$
\int_{I} \gamma_{k}(t) d t \leqslant[E=2] \frac{6}{q_{K+1}(x) F_{k-K}} h \log 1 / h+[E>2] \frac{72}{q_{K} F_{k-K}} h .
$$

\section{Démonstration}

Soit $\mathfrak{c}$ la cellule de profondeur $K$ qui contient $I$. Comme $\mathfrak{c}$ est l'intervalle d'extrémités

$$
\frac{p_{K}}{q_{K}} \quad \text { et } \frac{p_{K}+p_{K-1}}{q_{K}+q_{K-1}}
$$


nous pouvons écrire

$$
\begin{aligned}
x+(-1)^{K} h / 2 & =\frac{u p_{K}+p_{K-1}}{u q_{K}+q_{K-1}} \\
x+(-1)^{K-1} h / 2 & =\frac{v p_{K}+p_{K-1}}{v q_{K}+q_{K-1}},
\end{aligned}
$$

avec $1 \leqslant u<1 / \alpha_{K}(x)<v<+\infty$. Posons $m=\lfloor u\rfloor$ et $n=\lfloor v\rfloor$, de sorte que $1 \leqslant m \leqslant a_{K+1}(x) \leqslant n$ et $E=n-m+1, n>m$.

On a donc, en utilisant l'identité (9),

$$
\begin{aligned}
h & =\left|\frac{u p_{K}+p_{K-1}}{u q_{K}+q_{K-1}}-\frac{v p_{K}+p_{K-1}}{v q_{K}+q_{K-1}}\right| \\
& =\frac{v-u}{\left(u q_{K}+q_{K-1}\right)\left(v q_{K}+q_{K-1}\right)} \\
& \geqslant \frac{v-u}{q_{K}^{2}(u+1)(v+1)} \\
& \geqslant \frac{v-u}{6 q_{K}^{2} m n},
\end{aligned}
$$

où la dernière minoration découle de $u+1 \leqslant 3 m$ et $v+1 \leqslant 2 n$.

Rappelons que les cellules de profondeur $K+1$ incluses dans $\mathfrak{c}$ sont les intervalles

On a

$$
\mathfrak{c}_{\ell}=\left\{\frac{s p_{K}+p_{K-1}}{s q_{K}+q_{K-1}}, \ell<s<\ell+1\right\} \quad(\ell \geqslant 1) .
$$

$$
\begin{aligned}
x+(-1)^{K} h / 2 & \in \overline{\mathfrak{c}_{m}}, \\
x+(-1)^{K-1} h / 2 & \in \overline{\mathfrak{c}_{n}} .
\end{aligned}
$$

D'autre part, $I$ est inclus dans la réunion des $\overline{\mathfrak{c}_{\ell}}, m \leqslant \ell \leqslant n$, donc

$$
\int_{I} \gamma_{k}(t) d t \leqslant \sum_{n \leqslant \ell \leqslant m} \int_{\mathfrak{c}_{\ell}} \beta_{k-1}(t) \log \left(1 / \alpha_{k}(t)\right) d t .
$$

Distinguons alors deux cas.

Premier cas : $E=2$.

On a donc $n=m+1$. Comme $I \subset \overline{\mathfrak{c}_{m}} \cup \overline{\mathfrak{c}_{m+1}}$, on a

$$
q_{K+1}(t)=\left\{\begin{array}{l}
m q_{K}+q_{K-1} \\
\text { ou } \\
(m+1) q_{K}+q_{K-1}
\end{array} \quad\left(t \in I, t \notin \partial \mathfrak{c}_{m}\right)\right.
$$

En particulier, pour $t \in I, t \notin \partial \mathfrak{c}_{m}$, on a

$$
\frac{q_{K+1}(t)}{q_{K+1}(x)} \geqslant \frac{m q_{K}+q_{K-1}}{(m+1) q_{K}+q_{K-1}} \geqslant \frac{1}{2} .
$$

Par conséquent, d'après la majoration (21) et la proposition 2,

$$
\int_{I} \gamma_{k}(t) d t \leqslant \frac{2}{q_{K+1}(x) F_{k-K}} \int_{I} \log \left(1 / \alpha_{k}(t)\right) d t \leqslant \frac{2}{q_{K+1}(x) F_{k-K}} e h \log (1 / h) .
$$


Deuxième cas : $E \geqslant 3$.

On a donc $v-u \geqslant 1$, d'où

$$
n-m \leqslant v-(u-1) \leqslant 2(v-u) .
$$

D'après la majoration (21) appliquée avec $i=K$ et $j=k-K-1$ dans (41),

$$
\begin{aligned}
\int_{I} \gamma_{k}(t) d t & \leqslant \frac{1}{F_{k-K}} \sum_{n \leqslant \ell \leqslant m} \int_{\mathfrak{c}_{\ell}} \frac{\log \left(1 / \alpha_{k-K-1}\left(\alpha_{K+1}(t)\right)\right)}{q_{K+1}(t)} d t \\
& \leqslant \frac{1}{q_{K} F_{k-K}} \sum_{n \leqslant \ell \leqslant m} \frac{1}{\ell} \int_{\mathfrak{c}_{\ell}} \log \left(1 / \alpha_{k-K-1}\left(\alpha_{K+1}(t)\right)\right) d t
\end{aligned}
$$

où la dernière inégalité provient du fait que pour $t \in \mathfrak{c}_{\ell}, q_{K+1}(t)=\ell q_{K}+$ $q_{K-1}$. Nous effectuons dans chaque $\int_{\mathfrak{c}_{\ell}}$ le changement de variable $w=$ $\alpha_{K+1}(t)$. Notant que d'après (34)

$$
d t=(-1)^{K+1} \frac{d w}{\left(q_{K+1}+w q_{K}\right)^{2}}
$$

nous obtenons,

$$
\begin{aligned}
\int_{I} \gamma_{k}(t) d t & \leqslant \frac{1}{q_{K} F_{k-K}} \sum_{m \leqslant \ell \leqslant n} \frac{1}{\ell} \int_{0}^{1} \log \left(1 / \alpha_{k-K-1}(w)\right) \frac{d w}{\left(q_{K+1}+w q_{K}\right)^{2}} \\
& \leqslant \frac{1}{q_{K}^{3} F_{k-K}} \int_{0}^{1} \log \left(1 / \alpha_{k-K-1}(w)\right) d w \sum_{m \leqslant \ell \leqslant n} \frac{1}{\ell^{3}} \\
& \leqslant \frac{2}{q_{K}^{3} F_{k-K}} \int_{0}^{1} \log \left(1 / \alpha_{k-K-1}(w)\right) \frac{d w}{1+w} \sum_{m \leqslant \ell \leqslant n} \frac{1}{\ell^{3}} .
\end{aligned}
$$

En utilisant le lemme 3 et l'invariance de la mesure $\mu$ par $\alpha_{k-K-1}$, il vient

$$
\int_{I} \gamma_{k}(t) d t \leqslant \frac{6(n-m)}{q_{K}^{3} F_{k-K} m^{2} n} \int_{0}^{1} \log (1 / w) d w=\frac{6(n-m)}{q_{K}^{3} F_{k-K} m^{2} n},
$$

et, d'après (40) et (44),

$$
\int_{I} \gamma_{k}(t) d t \leqslant 12 \frac{v-u}{q_{K}^{3} F_{k-K} m^{2} n} \leqslant 72 \frac{h}{q_{K} F_{k-K} m} \leqslant 72 \frac{h}{q_{K} F_{k-K}} .
$$

La conclusion découle de (43) et (45).

Proposition 11. Pour $k \in \mathbb{N}$ tel que $k>K$ on $a$

$$
\begin{aligned}
& \int_{I} \gamma_{k}(t) d t \\
& \quad \leqslant \frac{h}{F_{k-K}}\left(\frac{72[E>2]}{q_{K}}+12 \frac{[E=2] \log q_{K+2}(x)}{q_{K+1}(x)}+12 \frac{[E=2] \log q_{K+3}(x)}{q_{K+2}(x)}\right) .
\end{aligned}
$$

\section{Démonstration}


On a

$$
\begin{aligned}
\frac{\log (1 / h)}{q_{K+1}(x)} & \leqslant \frac{\log \left(\left(2 \delta_{K+1}(x)\right)^{-1}\right)}{q_{K+1}(x)} \\
& \leqslant 2 \frac{\log q_{K+2}(x)}{q_{K+1}(x)}+2 \frac{\log q_{K+3}(x)}{q_{K+2}(x)}
\end{aligned}
$$

d'après la proposition 5 . Le résultat découle de cette inégalité et de la proposition 10 .

7. Comportement de $\Psi$ aU Voisinage D'un point Rationnel

Nous commençons par traiter les cas des points 0 et 1 .

Lemme 4. On a

$$
\int_{0}^{x} \Phi(\alpha(t)) d t=O(x) \quad(0 \leqslant x \leqslant 1) .
$$

\section{Démonstration}

Pour $0<x<1$, on a

$$
\begin{aligned}
\int_{0}^{x} \Phi(\alpha(t)) d t & \leqslant \int_{0}^{1 / a_{1}(x)} \Phi(\alpha(t)) d t \quad\left(\operatorname{car} x \leqslant 1 /\lfloor 1 / x\rfloor=1 / a_{1}(x)\right) \\
& =\sum_{n \geqslant a_{1}(x)} \int_{1 /(n+1)}^{1 / n} \Phi\left(\frac{1}{t}-n\right) d t \\
& =\sum_{n \geqslant a_{1}(x)} \int_{0}^{1} \Phi(u) \frac{d u}{(n+u)^{2}} \\
& =\int_{0}^{1} \Phi(u) \sum_{n \geqslant a_{1}(x)} \frac{1}{(n+u)^{2}} d u \\
& \leqslant \frac{2}{a_{1}(x)} \int_{0}^{1} \Phi(u) d u=O(x)
\end{aligned}
$$

Lemme 5. Pour $0<x \leqslant 1$, on $a$

$$
\Psi(x)=x \log (1 / x)+x+O\left(x^{2}\right),
$$

et

$$
\Psi(1)-\Psi(1-x)=x \log (1 / x)+x+O\left(x^{2} \log (2 / x)\right)
$$

\section{Démonstration}

Pour $0<x \leqslant 1$, on a, d'après l'équation fonctionnelle de $\Phi$,

$$
\begin{aligned}
\Psi(x) & =\int_{0}^{x} \Phi(t) d t=\int_{0}^{x}(t \Phi(\alpha(t))+\log (1 / t)) d t \\
& =\int_{0}^{x} \log (1 / t) d t+O\left(x \int_{0}^{x} \Phi(\alpha(t)) d t\right)=x \log (1 / x)+x+O\left(x^{2}\right),
\end{aligned}
$$

d'après le lemme 4 . 
Pour établir (47), il suffit de traiter le cas $0<x<1 / 2$. On a

$$
\begin{aligned}
\Psi(1)-\Psi(1-x) & =\int_{1-x}^{1} \Phi(t) d t=\int_{1-x}^{1}(t \Phi(\alpha(t))+\log (1 / t)) d t \\
& =\int_{1-x}^{1}(t \Phi((1-t) / t)+\log (1 / t)) d t
\end{aligned}
$$

En effectuant le changement de variable $u=(1-t) / t$ puis en employant (46), nous obtenons

$$
\begin{aligned}
\Psi(1)-\Psi(1-x) & =\int_{0}^{x /(1-x)}\left(\frac{\Phi(u)}{1+u}+\log (1+u)\right) \frac{d u}{(1+u)^{2}} \\
& =\int_{0}^{x /(1-x)}(\Phi(u)+O(u \Phi(u))+O(u)) d u \\
& =\frac{x}{1-x} \log \frac{1-x}{x}+\frac{x}{1-x}+O\left(x^{2} \log (1 / x)\right) \\
& =x \log (1 / x)+x+O\left(x^{2} \log (1 / x)\right) .
\end{aligned}
$$

Nous sommes maintenant en mesure d'établir le point (iii) du théorème 2 .

Proposition 12. On a uniformément pour $r$ rationnel et $h$ réel tels que $0<r<1, q^{2}|h|<2 / 3$ (où $q$ est le dénominateur de l'écriture irréductible de $r)$ :

$$
\Psi(r+h)-\Psi(r)=\frac{h}{q} \log (1 /|h|)+\left(\frac{1}{q}-2 \frac{\log q}{q}+\Phi(r)\right) h+O\left(q h^{2} \log \left(\left(q^{2}|h|\right)^{-1}\right)\right) .
$$

\section{Démonstration}

Soit $K \in \mathbb{N}^{*}$ la profondeur du nombre rationnel $r$. Comme $0<|h|<$ $2 / 3 q^{2}$, on sait d'après le $\S 5.3$ que l'intervalle ouvert d'extrémités $r$ et $r+h$ est inclus dans l'une des deux cellules de profondeur $K$ :

- $\mathfrak{c}$ d'extrémités $\frac{p-p_{K-1}}{q-q_{K-1}}$ et $r$;

- $\mathfrak{c}^{\prime}$ d'extrémités $r$ et $\frac{p+p_{K-1}}{q+q_{K-1}}$.

Les trois nombres $\frac{p-p_{K-1}}{q-q_{K-1}}, r$ et $\frac{p+p_{K-1}}{q+q_{K-1}}$ se succédent dans cet ordre si $K$ est pair, dans l'ordre inverse si $K$ est impair. Par conséquent,

$$
(-1)^{K} h>0 \Leftrightarrow r+h \in \mathfrak{c}^{\prime}
$$

On a

$$
\begin{aligned}
\Psi(r+h)-\Psi(r) & =\int_{r}^{r+h} \Phi(t) d t \\
& =\sum_{k<K} \int_{r}^{r+h} \gamma_{k}(t) d t+\int_{r}^{r+h} \beta_{K-1}(t) \Phi\left(\alpha_{K}(t)\right) d t
\end{aligned}
$$


d'après l'identité (8). Or,

$$
\begin{aligned}
\left|\int_{r}^{r+h} \sum_{k<K} \gamma_{k}(t) d t-h \Phi(r)\right| & =\left|\sum_{k<K} \int_{r}^{r+h}\left(\gamma_{k}(t)-\gamma_{k}(r)\right) d t\right| \\
& \leqslant \frac{3}{2} h^{2} \sum_{k<K} q_{k+1} \leqslant \frac{9}{2} q h^{2}
\end{aligned}
$$

d'après la proposition 7 et l'inégalité (13).

Il reste donc à établir

$$
\int_{r}^{r+h} \beta_{K-1}(t) \Phi\left(\alpha_{K}(t)\right) d t=\frac{1}{q} h \log \left(\left(q^{2}|h|\right)^{-1}\right)+\frac{h}{q}+O\left(q h^{2} \log \left(\left(q^{2}|h|\right)^{-1}\right)\right) .
$$

La fonction $\alpha_{K}$ est définie sur chacune des cellules $\mathfrak{c}$ et $\mathfrak{c}^{\prime}$. Suivant que $r+h$ appartienne à $\mathfrak{c}$ ou à $\mathfrak{c}^{\prime}$, nous prolongeons $\alpha_{K}$ (par continuité à droite ou à gauche suivant la parité de $K$ ) respectivement à $\mathfrak{c} \cup\{r\}$ ou $\mathfrak{c}^{\prime} \cup\{r\}$, et dans l'intégrale du premier membre de (49) nous effectuons le changement de variables $u=\alpha_{K}(t)$. Distinguons les deux cas.

- Premier cas : $(-1)^{K} h>0$. Dans ce cas, l'intervalle d'extrémités $r$ et $r+h$ est inclus dans $\overline{\mathfrak{c}^{\prime}}$. On a $q_{K}=q, p_{K}=p$ et

$$
u=\alpha_{K}(t)=\frac{q_{K} t-p_{K}}{-q_{K-1} t+p_{K-1}}
$$

de sorte que, compte tenu de (34),

$$
\int_{r}^{r+h} \beta_{K-1}(t) \Phi\left(\alpha_{K}(t)\right) d t=(-1)^{K} \int_{\alpha_{K}(r)}^{\alpha_{K}(r+h)} \Phi(u) \frac{d u}{\left(q_{K}+q_{K-1} u\right)^{3}}
$$

On a $\alpha_{K}(r)=0$ et

$$
\begin{aligned}
\alpha_{K}(r+h) & =\frac{q^{2} h}{-q_{K-1} p-q_{K-1} q h+p_{K-1} q} \\
& =\frac{q^{2}|h|}{1-|h| q q_{K-1}} .
\end{aligned}
$$

Nous posons donc

$$
x^{\prime}=\frac{q^{2}|h|}{1-|h| q q_{K-1}} .
$$


Comme $q_{K-1} \leqslant q / 2$, on a $0<x^{\prime}<1$ et

$$
\begin{aligned}
\int_{r}^{r+h} & \beta_{K-1}(t) \Phi\left(\alpha_{K}(t)\right) d t \\
& =\frac{(-1)^{K}}{q^{3}} \int_{0}^{x^{\prime}} \Phi(u) \frac{d u}{\left(1+u \frac{q_{K-1}}{q}\right)^{3}} \\
& =\frac{(-1)^{K}}{q^{3}} \int_{0}^{x^{\prime}} \Phi(u)(1+O(u)) d u \\
& =\frac{(-1)^{K}}{q^{3}} x^{\prime} \log \left(1 / x^{\prime}\right)+\frac{(-1)^{K}}{q^{3}} x^{\prime}+O\left(q^{-3} x^{\prime 2} \log \left(2 / x^{\prime}\right)\right)
\end{aligned}
$$

où la dernière égalité résulte de (46) et du lemme 4.

- Deuxième cas : $(-1)^{K} h<0$. Dans ce cas, l'intervalle d'extrémités $r$ et $r+h$ est inclus dans $\overline{\mathfrak{c}}$. Dans cette cellule, on a

$$
u=\alpha_{K}(t)=\frac{\left(q-q_{K-1}\right) t-p+p_{K-1}}{-q_{K-1} t+p_{K-1}}
$$

Par suite, $\alpha_{K}(r)=1$ et

$$
\begin{aligned}
\alpha_{K}(r+h) & =\frac{\left(q-q_{K-1}\right) \frac{p}{q}-\left(p-p_{K-1}\right)+h\left(q-q_{K-1}\right)}{-q_{K-1} \frac{p}{q}+p_{K-1}-q_{K-1} h} \\
& =1+\frac{h q^{2}}{(-1)^{K}-q q_{K-1} h}=1-x,
\end{aligned}
$$

avec cette fois

$$
x=\frac{q^{2}|h|}{1+|h| q q_{K-1}}(\leqslant 2 / 3) .
$$

Nous avons donc, d'après (47),

$$
\begin{aligned}
\int_{r}^{r+h} & \beta_{K-1}(t) \Phi\left(\alpha_{K}(t)\right) d t \\
& =(-1)^{K-1} \int_{1-x}^{1} \Phi(u) \frac{d u}{\left(q+(u-1) q_{K-1}\right)^{3}} \\
& =\frac{(-1)^{K-1}}{q^{3}} \int_{1-x}^{1} \Phi(u) d u+O\left(q^{-3} \int_{1-x}^{1} \Phi(u)(1-u) d u\right) \\
& =\frac{(-1)^{K-1}}{q^{3}} \int_{1-x}^{1} \Phi(u) d u+O\left(q^{-3} x^{2} \log (1 / x)\right) \\
& =\frac{(-1)^{K-1}}{q^{3}} x \log (1 / x)+\frac{(-1)^{K-1}}{q^{3}} x+O\left(q^{-3} x^{2} \log (1 / x)\right)
\end{aligned}
$$

Nous avons donc dans les deux cas, avec $y=x$ ou $x^{\prime}$, $\int_{r}^{r+h} \beta_{K-1}(t) \Phi\left(\alpha_{K}(t)\right) d t=\frac{\operatorname{sgn}(h)}{q^{3}} y \log (1 / y)+\frac{\operatorname{sgn}(h)}{q^{3}} y+O\left(q^{-3} y^{2} \log (2 / y)\right)$ 
Comme $q_{K-1}<q / 2$ et $|h|<2 / 3 q^{2}$, nous avons

$$
\log (1 / y)=\log \left(\left(q^{2}|h|\right)^{-1}\right)+O\left(q^{2} h\right),
$$

et

ce qui entraîne bien (49).

$$
y=q^{2}|h|+O\left(h^{2} q^{4}\right),
$$

\section{Comportement de $\Psi$ au voisinage D'un nombre de Cremer}

Nous commençons par démontrer le point $(i)$ du théorème 2 , qui concerne tous les irrationnels, qu'ils soient de Cremer ou de Brjuno.

Proposition 13. Soit $x \in X$. On a

$$
\Psi(x+h / 2)-\Psi(x-h / 2)=o(h \log (1 / h)) \quad(h \rightarrow 0) .
$$

\section{Démonstration}

Soit $x \in X$ et $K \in \mathbb{N}$. Soit $0<h<\min \left(e^{-2}, 2 \delta_{K}(x)\right)$ de sorte que l'intervalle $I=] x-h / 2, x+h / 2$ [ soit inclus dans la cellule de profondeur $K$ contenant $x$.

Nous avons, d'après les majorations (23) et (21),

$$
\int_{I} \Phi(t) d t \leqslant h \sum_{k<K} \frac{\log \left(2 q_{k+1}\right)}{q_{k}}+\sum_{k \geqslant K} \frac{1}{q_{K} F_{k-K+1}} \int_{I} \log \left(\alpha_{k}(t)\right) d t,
$$

avec $q_{k}=q_{k}(x)$ pour $k \leqslant K$. En employant la proposition 2 et l'inégalité (14), nous obtenons

$$
\int_{I} \Phi(t) d t \leqslant 4 h \log \left(2 q_{K}\right)+\frac{10}{q_{K}} h \log (1 / h) .
$$

Par conséquent,

$$
\limsup _{h \rightarrow 0} \frac{|\Psi(x+h / 2)-\Psi(x-h / 2)|}{h \log (1 / h)} \leqslant \frac{10}{q_{K}} .
$$

Comme $K$ est arbitraire, cela démontre le résultat.

Nous démontrons maintenant le point $(i i)$ du théorème 2 lorsque $x$ est un nombre de Cremer.

Proposition 14. Soit $x$ un nombre de Cremer. On a

$$
\lim _{h \rightarrow 0} \frac{\Psi(x+h)-\Psi(x)}{h}=+\infty .
$$

\section{Démonstration}

Comme la fonction $\gamma_{k}$ est positive, nous avons en toute généralité pour $h>0$,

$$
\begin{aligned}
\frac{\Psi(x+h)-\Psi(x)}{h} & =\frac{1}{h} \int_{x}^{x+h} \sum_{k \geqslant 0} \gamma_{k}(t) d t \geqslant \frac{1}{h} \int_{x}^{x+h} \sum_{k \leqslant K} \gamma_{k}(t) d t \\
& =\sum_{k \leqslant K} \frac{\int_{x}^{x+h} \gamma_{k}(t) d t}{h} .
\end{aligned}
$$


Si $x$ est irrationnel, chacune des fonctions $\gamma_{k}$ est continue au point $x$, ce qui entraîne

$$
\liminf _{h \rightarrow 0} \frac{\Psi(x+h)-\Psi(x)}{h} \geqslant \sum_{k \leqslant K} \gamma_{k}(x) \quad(x \in X, K \in \mathbb{N}) .
$$

Si $x$ est un point de Cremer, le membre de droite tend vers l'infini avec $K$, ce qui implique le résultat voulu.

\section{Démonstration du théorème 1 et COMportement de $\Psi$ AU VOISINAGE D'UN NOMBRE DE BRJUNO}

Les propositions 14 et 12 impliquent que tout point de Lebesgue de $\Phi$ est nécessairement un nombre de Brjuno. Il reste donc à établir que

$$
\frac{1}{h} \int_{x-h / 2}^{x+h / 2}|\Phi(t)-\Phi(x)| d t \rightarrow 0 \quad(h \rightarrow 0),
$$

quand $x$ est un nombre de Brjuno. Cela impliquera directement le point (ii) du théorème 2 lorsque $x_{0}$ est un nombre de Brjuno.

Par densité de $\mathbb{R} \backslash \mathbb{Q}$ dans $\mathbb{R}$, on peut supposer que les nombres $x \pm h / 2$ sont irrationnels. Posons alors $I=[x-h / 2, x+h / 2]$ et notons $K=K(x, h)$ la profondeur de ce segment. Nous avons

$$
\begin{aligned}
\int_{I}|\Phi(t)-\Phi(x)| d t \leqslant & \sum_{k<K} \int_{I}\left|\gamma_{k}(t)-\gamma_{k}(x)\right| d t+\int_{I} \gamma_{K}(t) d t \\
& +\sum_{k>K} \int_{I} \gamma_{k}(t) d t+h \sum_{k \geqslant K} \gamma_{k}(x) .
\end{aligned}
$$

Majorons chacun des quatre termes du second membre. En notant désormais $q_{j}=q_{j}(x)$ pour tout $j$, on a

$$
\begin{aligned}
\frac{1}{h} \sum_{k<K} \int_{I}\left|\gamma_{k}(t)-\gamma_{k}(x)\right| d t & \left.\leqslant \frac{3 h}{2} \sum_{k<K} q_{k+1} \quad \text { (d'après la proposition } 7\right) \\
& \leqslant \frac{9}{2} q_{K} h \quad(\text { d'après }(13)) \\
& \leqslant \frac{9}{q_{K+1}}
\end{aligned}
$$

la dernière inégalité résultant de la proposition 4 puisque $\left.h / 2<\delta_{K}(x)\right)$; puis,

$$
\begin{aligned}
\frac{1}{h} \int_{I} \gamma_{K}(t) d t & \leqslant 8 \gamma_{K}(x)+\frac{6 \log q_{K}+4}{q_{K}} \quad(\text { d'après la proposition } 9) \\
& \leqslant \frac{14 \log q_{K+1}+10}{q_{K}} \quad(\text { d'après }(23))
\end{aligned}
$$


ensuite, d'après la proposition 11,

$$
\begin{aligned}
\frac{1}{h} \sum_{k>K} \int_{I} \gamma_{k}(t) d t & \leqslant\left(\frac{72}{q_{K}}+12 \frac{\log q_{K+2}}{q_{K+1}}+12 \frac{\log q_{K+3}}{q_{K+2}}\right) \sum_{k>K} \frac{1}{F_{k-K}} \\
& \leqslant \frac{242}{q_{K}}+41 \frac{\log q_{K+2}}{q_{K+1}}+41 \frac{\log q_{K+3}}{q_{K+2}} ;
\end{aligned}
$$

et enfin

$$
\sum_{k \geqslant K} \gamma_{k}(x) \leqslant \sum_{k \geqslant K} \frac{\log \left(2 q_{k+1}\right)}{q_{k}} \quad \text { (d'après (23)). }
$$

Finalement, on obtient

$$
\frac{1}{h} \int_{I}|\Phi(t)-\Phi(x)| d t \leqslant \sum_{k \geqslant K} \frac{42 \log q_{k+1}+253}{q_{k}}
$$

Puisque $x$ est un nombre de Brjuno, le membre de droite de (53) tend vers 0 lorsque $K$ tend vers l'infini, et donc lorsque $h$ tend vers 0 d'après (31), ce qui achéve la preuve.

\section{DÉmonstration du théorème 3}

Le lemme $5 \mathrm{du} \S 7$ prouve que

$$
\omega(h) \geqslant h \log (1 / h)+O(h) .
$$

Il reste donc à établir

$$
\omega(h) \leqslant h \log (1 / h)+O(h) .
$$

Lorsque $h>e^{-2}$, il s'agit de montrer que $\omega(h)=O(1)$, ce qui est vrai car $\Phi \in L^{1}(0,1)$. Il suffit donc de montrer que si $I$ est un segment de longueur $h \leqslant e^{-2}$ inclus dans $[0,1]$ on a

$$
\int_{I} \Phi(t) d t \leqslant h \log (1 / h)+O(h) .
$$

En fait, par densité de $\mathbb{R} \backslash \mathbb{Q}$ dans $\mathbb{R}$, on peut également supposer que les extrémités et le milieu $x$ de $I$ sont irrationnels. Soit alors $K$ la profondeur de $I$ et $E$ son épaisseur.

Notons $\mathfrak{c}=\mathfrak{c}\left(a_{1}, \ldots, a_{K}\right)$ la cellule de profondeur $K$ qui contient $I$ et, pour $j \leqslant K$, désignons par $p_{j}$ et $q_{j}$ les valeurs constantes de ces fonctions dans c. Comme $I$ n'est pas inclus dans une cellule de profondeur $K+1$, il existe $a_{K+1} \geqslant 2$ tel que le nombre rationnel

$$
r=\left[0 ; a_{1}, \ldots, a_{K}, a_{K+1}\right]=\frac{p}{q} \quad((p, q)=1)
$$

appartienne à $I$. Observons que $p$ et $q$ sont les valeurs respectives des fonctions $p_{K+1}$ et $q_{K+1}$ dans la cellule $\mathfrak{c}\left(a_{1}, \ldots, a_{K}, a_{K+1}\right)$. D'autre part, si $E=2$, le nombre $x$ se situe soit dans la cellule $\mathfrak{c}\left(a_{1}, \ldots, a_{K}, a_{K+1}\right)$, soit dans la cellule $\mathfrak{c}\left(a_{1}, \ldots, a_{K}, a_{K+1}-1\right)$. Par conséquent

$$
q_{K+1}(x)=q \text { ou }\left(q-q_{K}\right) \text {, et donc } q_{K+1}(x) \geqslant q / 2 \text {. }
$$


Nous distinguons alors deux cas : $q \leqslant 100$ et $q>100$, où la valeur 100 est choisie pour obtenir simplement l'inégalité cherchée dans le second cas (pour le premier cas, n'importe quelle valeur convient).

Premier cas : $q \leqslant 100$.

Écrivons $I=[r-\alpha h, r+\beta h]$ avec $0<\alpha, \beta<1$ et $\alpha+\beta=1$. On peut supposer $h<1 / 15000$, et donc $q^{2} h<2 / 3$. D'après la proposition 12 , on a alors

$$
\begin{aligned}
\int_{I} \Phi(t) d t & =(\Psi(r+\beta h)-\Psi(r))+(\Psi(r)-\Psi(r-\alpha h)) \\
& =\frac{\beta h}{q} \log \left((\beta h)^{-1}\right)+\frac{\alpha h}{q} \log \left((\alpha h)^{-1}\right)+O(h) \\
& =\frac{h \log (1 / h)}{q}+\frac{h}{q}(\alpha \log (1 / \alpha)+\beta \log (1 / \beta))+O(h) \\
& \leqslant h \log 1 / h+O(h),
\end{aligned}
$$

car la fonction $t \mapsto t \log (1 / t)$ est bornée sur l'intervalle [0,1].

Deuxième cas : $q>100$.

En notant $m$ le plus grand entier inférieur ou égal à $K$ tel que $q_{m} \leqslant 100$, nous effectuons la décomposition

$$
\int_{I} \Phi(t) d t=\sum_{k<m} \int_{I} \gamma_{k}(t) d t+\int_{I} \gamma_{m}(t) d t+\sum_{k>m} \int_{I} \gamma_{k}(t) d t
$$

Nous avons, d'après (23),

$$
\sum_{k<m} \int_{I} \gamma_{k}(t) d t \leqslant h \sum_{k<m} \frac{\log \left(2 q_{k+1}\right)}{q_{k}} \leqslant h \log \left(2 q_{m}\right) \sum_{k \geqslant 1} \frac{1}{F_{k}} \leqslant 3,36 h \log 200
$$

et donc

$$
\int_{I} \Phi(t) d t \leqslant \int_{I} \gamma_{m}(t) d t+\sum_{k>m} \int_{I} \gamma_{k}(t) d t+O(h) .
$$

Il reste à établir la majoration

$$
\int_{I} \gamma_{m}(t) d t+\sum_{k>m} \int_{I} \gamma_{k}(t) d t \leqslant h \log (1 / h)+O(h)
$$

Pour ce faire, nous distinguons deux sous-cas.

Premier sous-cas : $m<K$.

La fonction $q_{m+1}$ est alors constante sur $I$ et, par maximalité de $m$, on a $q_{m+1}>100$. Nous avons alors directement avec (21) et la proposition 2

$$
\begin{aligned}
\sum_{k>m} \int_{I} \gamma_{k}(t) d t & \leqslant \frac{1}{q_{m+1}} \sum_{k>m} \frac{1}{F_{k-m}} \int_{I} \log \left(1 / \alpha_{k}(t)\right) d t \\
& \leqslant \frac{3,36 e h \log (1 / h)}{100} \leqslant \frac{h}{4} \log (1 / h) .
\end{aligned}
$$

Par ailleurs la proposition 6 nous donne

$$
\int_{I} \gamma_{m}(t) d t \leqslant \frac{h \log (1 / h)}{2}+h .
$$


On obtient donc

$$
\int_{I} \Phi(t) d t \leqslant \frac{3}{4} h \log (1 / h)+O(h) .
$$

Deuxième sous-cas : $m=K$.

Nous appliquons alors les propositions 8 et 10 :

$$
\begin{aligned}
\int_{I} \gamma_{K}(t) d t+ & \sum_{k>K} \int_{I} \gamma_{k}(t) d t \\
\leqslant & h \log 1 / h\left(\frac{[E>2]+[E=2] / 2}{q_{K}}+\frac{6[E=2]}{q_{K+1}(x)} \sum_{k>K} \frac{1}{F_{k-K}}\right) \\
& +h\left(\frac{1}{q_{K}}+\frac{72[E>2]}{q_{K}} \sum_{k>K} \frac{1}{F_{k-K}}\right)
\end{aligned}
$$

Comme

$$
\begin{aligned}
\frac{[E>2]+[E=2] / 2}{q_{K}} & +\frac{6[E=2]}{q_{K+1}(x)} \sum_{k>K} \frac{1}{F_{k-K}} \\
& \leqslant[E>2]+[E=2]\left(\frac{1}{2}+\frac{12}{q} 3,36\right) \quad \text { (d'après (56)) } \\
& \leqslant 1 \quad(\operatorname{car} q>100),
\end{aligned}
$$

nous avons bien établi (55) dans tous les cas.

\section{Questions}

Il serait d'abord intéressant de déterminer le comportement asymptotique du module de continuité $L^{1}$ de la fonction de Brjuno,

$$
\omega_{1}(h)=\sup _{0<h^{\prime} \leqslant h} \int_{0}^{1}\left|\Phi\left(t+h^{\prime}\right)-\Phi(t)\right| d t
$$

(où $\Phi$ est prolongée par 1-périodicité sur $\mathbb{R}$ ), quand $h$ tend vers 0 .

D'autre part, dans [9], Marmi, Moussa et Yoccoz prolongent la fonction de Brjuno au demi-plan $\Im z>0$. Ils étudient en détail les propriétés de la fonction de Brjuno complexe, et notamment son comportement quand $\Im z \rightarrow 0$. Il serait intéressant de relier nos résultats et les leurs, en particulier le point (iii) du théorème 2 avec le $\$ 5.2 .9$ de [9]. Comme nous avons conçu le présent travail dans le cadre strict de l'analyse réelle, nous laissons ces questions ouvertes. 


\section{RÉFÉRENCES}

[1] P. Billingsley - Ergodic theory and information, John Wiley \& Sons Inc., New York, 1965.

[2] K. DAJAni \& C. KRAAIKAmP - Ergodic theory of numbers, Carus Mathematical Monographs, vol. 29, Mathematical Association of America, Washington, DC, 2002.

[3] W. Doeblin - «Remarques sur la théorie métrique des fractions continues », Compositio Math. 7 (1940), p. 353-371.

[4] A. M. D'YachKov - «Description of sets of Lebesgue points and summability points of a Fourier series », Mat. Sb. 182 (1991), p. 1367-1374.

[5] C. F. Gauss - Werke. Zehnten Bandes erste Mitteilung., Herausg. von der Kgl. Ges d. Wiss. zu Göttingen, B. G. Teubner, Leipzig, 1917.

[6] A. Y. Khinchin - Continued fractions, Dover, Mineola, 1997, Reprint of the 1964 translation.

[7] R. O. Kuzmin - «Sur un problème de Gauss », Atti Congr. Intern. Bologna 6 (1928), p. 83-89.

[8] S. Marmi, P. Moussa \& J.-C. YocCOZ - « The Brjuno functions and their regularity properties », Comm. Math. Phys. 186 (1997), p. 265-293.

[9] S. Marmi, P. Moussa \& J.-C. YocCOZ - «Complex Brjuno functions », J. Amer. Math. Soc. 14 (2001), p. 783-841.

[10] M. MEndÈs FrAnCE - «Remarks and problems on finite and periodic continued fractions », Enseign. Math. (2) 39 (1993), p. 249-257.

[11] I. Niven, H. S. Zuckerman \& H. L. Montgomery - An introduction to the theory of numbers, fifth ed., John Wiley \& Sons Inc., New York, 1991.

[12] C. RYll-NARDZEWSKI - « On the ergodic theorems. II. Ergodic theory of continued fractions », Studia Math. 12 (1951), p. 74-79.

[13] R. L. WheEden \& A. Zygmund - Measure and integral, an introduction to real analysis, Marcel Dekker Inc., New York, 1977.

[14] J.-C. YocCOZ - « Théorème de Siegel, nombres de Bruno et polynômes quadratiques », Astérisque 231 (1995), p. 3-88, Petits diviseurs en dimension 1.

BALAZARD, Michel

Institut de Mathématiques de Luminy, Laboratoire de Mathématiques Pures et UMR 6206

CNRS, Aix Marseille Université

Campus de Luminy, Case 907

13288 Marseille Cedex 9

FRANCE

Adresse électronique :

balazard@iml.univ-mrs.fr
MARTIN, Bruno

Appliquées

CNRS, Université du Littoral Côte d'Opale

50 rue F. Buisson, BP 599

62228 Calais Cedex

FRANCE

Adresse électronique :

martin@lmpa.univ-littoral.fr 